Acta Crystallographica Section B

Structural

Science

ISSN 0108-7681

Notes for Authors

major areas of research interest. They are always commissioned by the Section Editor, on the advice of the Editorial Board. Suggestions for suitable topics and of potential author(s) are welcomed by the Section Editor for discussion with the Board.

The Editor will discuss the treatment of the topic, the length of the Article and the delivery date of the manuscript with invited author(s). Lead Articles will be refereed in the normal manner. solve problems in the structural domain. Section $B$ has broad chemical coverage, encompassing metals and alloys, inorganics and minerals, metal-organics and purely organic compounds. Biological, particularly macromolecular, structural science is now covered by Section $D$. A more complete definition of scientific scope is given in an Editorial [Acta Cryst. (1994), B50, 1-3].

\section{Categories of contributions}

Contributions should conform to the general editorial style of the journal.

\subsection{Research Papers}

Full-length Research Papers should not normally exceed 15 journal pages (about 15000 words).

\subsection{Short Communications}

Short Communications are intended for the presentation of topics of limited scope, or for preliminary announcements of novel research findings. They are not intended for interim reports of work in progress, and must report results that are of scientific value in their own right. In Section $B$, it is unlikely that reports of individual structure determinations will be acceptable as Short Communications, since Section $C$ of Acta Crystallographica exists for this purpose.

Short Communications should not exceed two journal pages (about 1500 words). A maximum of two figures and two tables of appropriate size are permitted.

All Short Communications should be submitted to the Section Editor.

\subsection{Lead Articles}

Lead Articles are authoritative, comprehensive and forward-looking reviews of

\subsection{Topical Reviews}

A Topical Review is a short, highly focused survey covering a relatively narrow area of current research interest. It should not aim to be comprehensive, but a brief introduction should provide historical perspective and a brief conclusion should indicate likely future directions.

Topical Reviews will be limited to about ten journal pages (10 000 words) except in special agreed circumstances. Shorter reviews on rapidly evolving areas are also actively encouraged. They will be commissioned by the Section Editor either personally, or following a formal proposal by prospective author(s). Topical Reviews will be refereed in the normal way.

\subsection{Letters to the Editor}

These may deal with non-technical aspects of crystallography, its role, its propagation, the proper function of its Societies etc., or may make a technical observation that would usefully be brought to a wider audience. Letters should be sent to the Section Editor or to the Editor-inChief of Acta Crystallographica only.

\subsection{New Commercial Products}

Announcements of new commercial products are published free of charge. The descriptions, up to 300 words or the equivalent if a figure is included, should give the manufacturer's full address.

\section{Submission and handling of manuscripts}

\subsection{Submission}

Manuscripts and figures should be prepared using the file formats listed in $\$ 3.8$. Three paper copies and the electronic file(s) should be submitted; authors are reminded to keep an exact copy of the submission for later editorial adjustments and for checking proofs. Crystal data should be submitted in CIF format (see §11). Unless stated otherwise in $\$ 2$, the submission should be sent to the Section Editor or any of the Co-editors. On acceptance, an electronic version of the final manuscript will be required by the Editorial Office.

Contact details for the editors are available at http://journals.iucr.org/b/services/ editors.html

\subsection{Languages of publication}

Acta Crystallographica Section B will publish papers in English, French, German and Russian.

\subsection{Handling of manuscripts}

All contributions will be seen by referees (normally two) before they can be accepted for publication. The editor to whom the manuscript is sent is responsible for choosing referees and for accepting or rejecting the paper. This responsibility includes decisions on the final form of the paper and interpretation of these Notes when necessary.

If changes to a manuscript requested by the editorial staff or the Co-editor are not received within two months of transmittal to the author, the submission will automatically be withdrawn. Any subsequent communication of the material will be treated as a new submission in the editorial process.

For accepted papers, it is the responsibility of the Managing Editor to prepare the paper for printing. This may involve correspondence with the authors and/or the responsible editor in order to resolve ambiguities or to obtain satisfactory figures or tables. The date of acceptance that will appear on the published paper is the date on which the Managing Editor receives the last item required. Correspondence will be sent 
to the author who signed the letter of submission unless the Managing Editor is informed of some other suitable arrangement.

On rare occasions an editor may consider that a paper is better suited to a section of Acta Crystallographica other than that specified by the author(s), to the Journal of Applied Crystallography or to the Journal of Synchrotron Radiation. Any change to the section or journal of publication will only be made after full discussion with the communicating author.

\subsection{Author's warranty}

The submission of a paper is taken as an implicit guarantee that the work is original, that it is the author(s) own work, that all authors concur with and are aware of the submission, that all workers involved in the study are listed as authors or given proper credit in the acknowledegments, that the manuscript has not already been published (in any language or medium), and that it is not being considered and will not be offered elsewhere while under consideration for an IUCr journal.

\subsection{Copyright}

Except as required otherwise by national laws, an author must sign and submit a copy of the Transfer of Copyright Agreement form for each manuscript before it can be accepted.

\subsection{Author grievance procedure}

An author who believes that a paper has been unjustifiably treated by the Co-editor may appeal initially to the Section Editor and then to the Editor-in-Chief if still aggrieved by the decision.

\subsection{Contact e-mail address}

The contact author must provide an email address for editorial communications and despatch of electronic proofs.

\subsection{File format, naming and transfer}

The manuscript should be prepared using TEX, LATEX or Word. Authors are encouraged to use the templates available from the Editorial Office by e-mail (med@iucr.org) or by ftp (from the 'templates' directory). All Word submissions should be accompanied by an RTF (rich text format) file.

Figures may be sent in PostScript, encapsulated PostScript or TIFF formats. The resolution of bitmap graphics should be a minimum of 1200 d.p.i.
All files need to be given a filename constructed from the reference number supplied by the Co-editor. Files containing text in $\mathrm{T}_{\mathrm{E}} \mathrm{X}$ or $\mathrm{LT}_{\mathrm{E}} \mathrm{X}$ should be given the extension .tex, Word files should be given the extension .doc and RTF files .rtf. Illustrations in PostScript, encapsulated PostScript or TIFF format should be given the extensions .ps, .eps or .tif, respectively. Multiple files for the same submission should be identified by filenames constructed as ref.id.ext where id indicates the contents, e.g. xz1087.fig1.ps and xz1087.fig2.ps.

After acceptance, the final version of the paper should be sent to the Editorial Office by e-mail (med@iucr.org), on diskette or (for files larger than $70 \mathrm{~K}$ bytes) by ftp as described below.

\begin{tabular}{lc}
\hline (i) On your workstation enter: & ftp ftp.iucr.org \\
(ii) Wait for Name...: prompt & anonymous \\
and enter: & your e-mail \\
(iii) Wait for Password: & address \\
prompt and enter: & incoming/b \\
(iv) Wait for ftp> prompt & put j29.ps \\
and enter: & ja0325.ps \\
(v) Transfer a file from your & \\
account $(e . g$. j29.ps) as an & \\
identifiable name (e.g. & \\
ja0325.ps): & \\
(vi) Wait for ftp> prompt before sending another file \\
(vii) Finish off the ftp session \\
by entering: \\
(viii) Send an e-mail to Chester (bpub@iucr.org) \\
with a list of the files transferred by ftp.
\end{tabular}

\section{Abstract and synopsis}

All contributions must be accompanied by an English language Abstract and a one or two sentence Synopsis of the main findings of the paper for inclusion in the Table of Contents for the relevant issue. The Abstract should state as specifically and as quantitatively as possible the principal results obtained.

The Abstract should be suitable for reproduction by abstracting services without change in wording. It should not repeat information given in the title. Ordinarily 200 words suffice for Abstracts of Research Papers, Lead Articles and Topical Reviews, and 100 words for shorter contributions. It should make no reference to tables, diagrams, atom numbers or formulae contained in the paper. It should not contain footnotes. Numerical information given in the Abstract should not be repeated in the text. It should not include the use of 'we' or 'I'.

Literature references in an Abstract are discouraged. If a reference is unavoidable, it should be sufficiently full within the $A b s t r a c t$ for unambiguous identification, e.g. [Filippini (1990). Acta Cryst. B46, 643-645].

\section{Diagrams and photographs ('figures')}

Figures should be prepared using one of the file formats listed in $\$ 3.8$. Hard-copy figures must be provided in all cases.

The choice of tables and figures should be optimized to produce the shortest printed paper consistent with clarity. Duplicate presentation of the same information in both tables and figures is to be avoided, as is redundancy with the text.

In papers reporting novel organic or metal-organic structures, it is mandatory that a chemical structural diagram be included for each compound. Further, it is preferred that distances and angles are supplied in CIF format (see §11) for presentation in tabular form.

In a charge-density paper only those figures which are strictly necessary to illustrate the techniques or results described will be published: any others will be deposited. The text should be adequate to give the remaining information.

In papers which use powder profile fitting or refinement (Rietveld) methods, figures which present the experimental and calculated diffraction profiles of the material studied should also contain the difference profile. As primary diffraction data cannot be satisfactorily extracted from such figures, the basic digital diffraction data should be deposited (see $§ 12.3$ ).

\subsection{Quality}

Hard-copy greyscale or colour images should be provided as glossy prints; laser printer or photocopier output will generally be unsatisfactory for reproduction of such diagrams. High-resolution laser printer output is satisfactory for line drawings.

\subsection{Size}

Diagrams should be as small as possible consistent with legibility. They will normally be sized so that the greatest width including lettering is less than the width of a column in the journal.

\subsection{Lettering and symbols}

Fine-scale details and lettering must be large enough to be clearly legible (ideally $1.5-3 \mathrm{~mm}$ in height) after the whole diagram has been reduced to one column width. 
Lettering should be kept to a minimum; descriptive matter should be placed in the legend.

\subsection{Numbering and legends}

Diagrams should be numbered in a single series in the order in which they are referred to in the text. A list of the legends ("figure captions') should be included in the manuscript.

\subsection{Stereofigures}

Atom labelling when included should be on both left and right views in stereo perspective. Both views should be incorporated into a single figure.

\subsection{Colour figures}

Figures in colour are accepted at no cost to the author provided that the editor agrees that they improve the understanding of the paper.

\section{Tables}

Tables produced in Word should be prepared using the Word table editor.

\subsection{Economy in use of tables}

Numerical information is generally most economically presented in tables. Text and diagrams should not be redundant with the tables.

Structure factors, anisotropic displacement parameters, least-squares planes and unrefined $\mathrm{H}$-atom coordinates are usually deposited as electronic files, see $§ 11$.

\subsection{Design, numbering and size}

Tables should be numbered in a single series of arabic numerals in the order in which they are referred to in the text. They should be provided with a caption.

Tables should be carefully designed to occupy a minimum of space consistent with clarity.

\section{Mathematics and letter symbols}

Authors submitting in Word should use the Word equation editor to prepare displayed mathematical equations.

The use of the stop (period) to denote multiplication should be avoided except in scalar products. Generally no sign is required but, when one is, a multiplication sign $(x)$ should be used.

Vectors should be in bold type and tensors should be in bold-italic type.
Greek letters should not be spelled out.

Care should be taken not to cause confusion by using the same letter symbol in two different meanings.

Gothic, script or other unusual lettering should be avoided. Another typeface may be substituted if that used by the author is not readily available.

Equations, including those in published Appendices, should be numbered in a single series.

\section{Multimedia}

Multimedia additions to a paper (e.g. timelapse sequences, three-dimensional structures) are welcomed; they will be made available via Crystallography Journals Online.

\section{Nomenclature}

\subsection{Crystallographic nomenclature}

Authors should follow the general recommendations produced by the IUCr Commision on Crystallographic Nomenclature (see reports at http://www.iucr.org/ iucr-top/comm/cnom/).

Atoms of the same chemical species within an asymmetric unit should be distinguished by an appended arabic numeral. Chemical and crystallographic numbering should be in agreement wherever possible. When it is necessary to distinguish crystallographically equivalent atoms in different asymmetric units the distinction should be made by lower-case roman numeral superscripts (i.e. i, ii, iii etc.) to the original atom labels.

Space groups should be designated by the Hermann-Mauguin symbols. Standard cell settings, as listed in Volume A of International Tables for Crystallography, should be used unless objective reasons to the contrary are stated. When a non-standard setting is used, the list of equivalent positions should be given. Hermann-Mauguin symbols should also be used for designating point groups and molecular symmetry. It is helpful if the origin used is stated explicitly where there is a choice.

The choice of axes should normally follow the recommendations of the Commission on Crystallographic Data [Kennard et al. (1967). Acta Cryst. 22, 445-449].

A symbol such as 123 or $h k l$ without brackets is understood to be a reflection, (123) or $(h k l)$ a plane or set of planes, [123] or $[u v w]$ a direction, $\{h k l\}$ a form and $\langle u v w\rangle$ all crystallographically equivalent directions of the type $[u v w]$. Other bracket notations should be explicitly defined.

\subsection{Nomenclature of chemical compounds etc.}

Names of chemical compounds and minerals are not always unambiguous. Authors should therefore quote the chemical formulae, including chemical structural diagrams for organic and metalorganic compounds, of the substances dealt with in their papers.

Chemical formulae and nomenclature should conform to the rules of nomenclature established by the International Union of Pure and Applied Chemistry (IUPAC), the International Union of Biochemistry and Molecular Biology (IUBMB), the International Mineralogical Association and other appropriate bodies. As far as possible the crystallographic nomenclature should correspond to the systematic name.

Any accepted trivial or non-systematic name may be retained, but the corresponding systematic (IUPAC) name should also be given.

\subsection{Units}

The International System of Units (SI) is used except that the ångström (symbol $\AA$, defined as $10^{-10} \mathrm{~m}$ ) is generally preferred to the nanometre $(\mathrm{nm})$ or picometre $(\mathrm{pm})$ as the appropriate unit of length. Recommended prefixes of decimal multiples should be used rather than ' $\times 10^{n}$.

\section{References}

References to published work must be indicated by giving the authors' names followed immediately by the year of publication, e.g. Neder \& Schulz (1998) or (Neder \& Schulz, 1998). Where there are three or more authors the reference in the text should be indicated in the form Smith et al. (1998) or (Smith et al., 1998) etc. (all authors should be included in the full list).

At the end of the paper a list giving full details of all references should be appended separately. In the reference list, entries for journals [abbreviated in the style of Chemical Abstracts (the abbreviations Acta Cryst., J. Appl. Cryst. and J. Synchrotron Rad. are exceptions)], books, multi-author books, computer programs, personal communications and undated documents should be arranged alphabetically and conform with the following style:

Andrews, M., Wright, H. \& Clarke, S. A. (2000). In preparation. 
Bricogne, G. (1993). Acta Cryst. D49, 37-60. Carter, C. W. Jr (1990). Methods: a Companion to Methods in Enzymology, Vol. 1, pp. 12-24. New York: Academic Press.

Collaborative Computational Project, Number 4 (1994). Acta Cryst. D50, 760-763.

Crowther, R. A. (1972). The Molecular Replacement Method, edited by M. G. Rossmann, pp. 173-178. New York: Gordon and Breach.

International Union of Crystallography (2000). (IUCr) Crystallography Journals Online, http://journals.iucr.org.

Jancarik, J. \& Kim, S.-H. (1991). J. Appl. Chem. 24, 409-411.

Olsnes, S. \& Pihl, A. (1982). Molecular Action of Toxins and Viruses, edited by P. Cohen \& S. Van Heyningen, pp. 51-105. New York: Elsevier.

Stanlow, D. J. (2000). Acta Cryst. B56. In the press.

Strong, R. K. (1990). PhD thesis, Harvard University, USA.

Vogel, A. (1978). Textbook of Practival Organic Chemistry, 4th ed. London: Longman.

Wang, B.-C. (1985). Methods Enzymol. 115, 90112.

Yariv, J. (1983). Personal communication.

Note that inclusive page numbers must be given.

\section{Crystal structure determinations}

\subsection{Information requirements}

Papers which report the results of crystal structure determinations of small molecules or inorganic compounds must report the associated experimental data as required in the Notes for Authors for Section C of Acta Crystallographica. These data should be supplied as an electronic file in CIF format. The CIF will be used to generate standard tables of experimental details, atomic coordinates and selected geometric data; nonstandard geometric tables must either be encoded within the CIF or supplied as part of the manuscript (see $\$ 11.2$ ). Authors of powder diffraction papers should consult the notes provided at http://www.iucr.org/iucrtop/journals/acta/c/docs/powdersub.html.

For a checklist for the description of incommensurate modulated crystal structures see Chapuis et al. [Acta Cryst. (1997), A53, 95-100].

\subsection{CIF submissions}

To avoid delays in editorial handling, authors are encouraged to submit their CIF in the following way. (a) Before submission, the crystal data should be pre-checked by e-mailing the CIF to checkcif@iucr.org. A check report will be returned automatically to the sender's e-mail address. (b) Wherever possible, a PostScript printout of the standard tables that will be generated from the CIF should be obtained by sending the CIF (after completing the pre-check) to printb@iucr.org. This will return a PostScript file for local printing. Authors should mark on the printout which of the tables they wish to include in their manuscript along with any modifications required (for example, changes in the automatic numbering of the tables) and include the printout with the manuscript. (c) The manuscript accompanied by a CIF should then be submitted to the Co-editor. $(d)$ As soon as the contact author has received acknowledgement of their submission from the Co-editor, the CIF should be e-mailed to bpub@iucr.org, giving the manuscript number from the acknowledgement letter in the 'subject line', or in the publ_contact_letter field of the CIF.

While the manuscript is being reviewed by the Co-editor, the data in the CIF will be checked in Chester for completeness and internal consistency as described in the Notes for Authors for Section C of Acta Crystallographica. Any queries raised by the check procedures will be communicated to the authors via the Co-editor.

Any amendments to the crystal data during the review of the manuscript should be marked clearly on the printout generated from the original CIF. This document will be used by the editorial staff to update and revise the crystal data to be published as part of the accepted paper. However, if major revisions have been carried out since the CIF was sent for checking, it may be more appropriate to supply a new CIF with the accepted manuscript. This should be indicated clearly on the manuscript.

\section{Supplementary publication procedure (deposition)}

\subsection{Purpose and scope}

Parts of some papers are of interest to only a small number of readers, and the cost of printing these parts is not warranted. Arrangements have therefore been made for such material to be deposited with the IUCr electronic archive, with the Protein Data Bank, the Nucleic Acid Database and the ICDD as appropriate.

\subsection{IUCr electronic archive}

All material for deposition in the IUCr electronic archive should be supplied electronically.

Non-structural information, which may include:

details of the experimental procedure;

details of the stages of structure refinement;

details of mathematical derivations given only in outline in the main text and in mathematical Appendices;

lengthy discussion of points that are not of general interest or that do not lead to definite conclusions but that do have significant value;

additional diagrams,

should be supplied in one of the formats given in $\$ 3.8$.

Structural information (for small-molecule structures) should be supplied in CIF format; structure factors should be supplied as .fcf files.

\subsection{Powder diffraction data}

For papers that present the results of powder diffraction profile fitting or refinement (Rietveld) methods, the primary diffraction data, i.e. the numerical intensity of each measured point on the profile as a function of scattering angle, will be deposited.

\section{Crystallography Journals Online}

Full details of author services can be found at http://journals.iucr.org/b/services/authorservices.html.

\subsection{Electronic status information}

Authors may obtain information about the current status of their papers at http:// journals.iucr.org/services/status.html.

\subsection{Proofs}

Proofs will be provided electronically in portable document format (pdf). The correspondence author will be notified by email when the proofs are ready for downloading.

\subsection{Reprints}

After publication, the correspondence author will be able to download the electronic reprint of the published article, free of charge. Authors will also be able to order printed reprints at the proof stage. 


\section{International Union of Crystallography Transfer of Copyright Agreement}

Title of Article (Please type or use capital letters)

Authors (Please type or use capital letters)

Copyright to the above article is hereby transferred (except as otherwise required by national laws $\dagger$ ) to the International Union of Crystallography, effective if and when the article is accepted for publication in Acta Crystallographica, Journal of Applied Crystallography or Journal of Synchrotron Radiation. In addition to reproduction in conventional printed form your article will be stored electronically. Your assignment of Copyright signifies your agreement to the journal making arrangements to include your paper in document delivery services, CD-ROMs and electronic databases, etc., worldwide. This transfer of copyright also applies to data submitted in machine-readable form. However, the authors reserve the following:

(1) All proprietary rights other than copyright, such as patent rights.

(2) The right to use all or part of this article in future works prepared by or on the behalf of the author.

(3) In the case of 'work for hire', the right of the employer to incorporate all or part of this article in future works.

(4) The royalty-free right of the employer to make copies of this article, without further permission, for his or her own use but not for resale. Any identification or notice appearing on the pages to be reproduced must not be deleted.

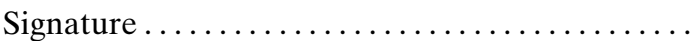

Name and position, if not author
Signature $\ldots \ldots \ldots \ldots \ldots \ldots \ldots \ldots \ldots \ldots \ldots \ldots \ldots \ldots \ldots \ldots \ldots \ldots \ldots$

Name and position, if not author

Date

Date

This Transfer of Copyright Agreement must be signed by at least one of the authors (who agrees to inform the others, if any) or, in the case of a 'work made for hire', by the employer.

By signing this form you certify that your contribution is your original work, has not been published before (in any language or medium) and is not being considered for publication elsewhere; that you have obtained permission for and acknowledged the source of any excerpts from other copyright works; and that to the best of your knowledge your paper contains no statements which are libellous, unlawful or in any way actionable.

The signed statement must be received before the article can be accepted for publication. Requests for further information should be sent to the Executive Secretary of the Union.

$\dagger$ In this case please give further details overleaf.

For use of the International Union of Crystallography only.

\begin{tabular}{|l|l|l|l|}
\hline M. E. Reference & Coeditor No. & Issue & Journal \\
& & & \\
\hline
\end{tabular}

This form may be photocopied. 